\title{
Scalloped and Yorkie are required for cell cycle re-entry of quiescent cells after tissue damage
}

\author{
Joy H. Meserve ${ }^{1}$ and Robert J. Duronio ${ }^{1,2,3,4, *}$
}

\begin{abstract}
Regeneration of damaged tissues typically requires a population of active stem cells. How damaged tissue is regenerated in quiescent tissues lacking a stem cell population is less well understood. We used a genetic screen in the developing Drosophila melanogaster eye to investigate the mechanisms that trigger quiescent cells to re-enter the cell cycle and proliferate in response to tissue damage. We discovered that Hippo signaling regulates compensatory proliferation after extensive cell death in the developing eye. Scalloped and Yorkie, transcriptional effectors of the Hippo pathway, drive Cyclin E expression to induce cell cycle re-entry in cells that normally remain quiescent in the absence of damage. Ajuba, an upstream regulator of Hippo signaling that functions as a sensor of epithelial integrity, is also required for cell cycle re-entry. Thus, in addition to its well-established role in modulating proliferation during periods of tissue growth, Hippo signaling maintains homeostasis by regulating quiescent cell populations affected by tissue damage.
\end{abstract}

KEY WORDS: Drosophila, Cell cycle, Quiescence, Apoptosis, Compensatory proliferation, Regeneration, Hippo signaling

\section{INTRODUCTION}

Tissue regeneration has fascinated biologists for many years, but the molecular mechanisms underlying this process have only recently begun to be understood. Pioneering experiments on regenerating hydra by the Abbé Trembley in the 1700s and on other organisms in the 1800s (Morgan, 1901) established that regeneration functions as a reparative process to replace tissues damaged by injury or disease and occurs as a restorative process to repair old and damaged tissues throughout an organism's life. Although early experiments on highly regenerative animals, such as hydra, are fundamental for our understanding of regeneration, the lack of facile genetic manipulation in these organisms made identifying molecular mechanisms difficult.

In the 1970s, Drosophila melanogaster emerged as a powerful and genetically tractable experimental system in which to study regeneration. Larval imaginal discs, which are epithelial tissues that proliferate during larval life and differentiate during pupation into adult structures, are able to regrow after substantial tissue loss due to irradiation or removal of fragments by surgery (Bryant, 1971; Haynie and Bryant, 1977; Worley et al., 2012). In these imaginal discs, a process called compensatory proliferation $(\mathrm{CP})$ replaces

\footnotetext{
${ }^{1}$ Curriculum in Genetics \& Molecular Biology, University of North Carolina, Chape Hill, NC 27599, USA. ${ }^{2}$ Integrative Program for Biological and Genome Sciences, University of North Carolina, Chapel Hill, NC 27599, USA. ${ }^{3}$ Departments of Biology and Genetics, University of North Carolina, Chapel Hill, NC 27599, USA.

${ }^{4}$ Lineberger Comprehensive Cancer Center, University of North Carolina, Chapel Hill, NC 27599, USA

*Author for correspondence (duronio@med.unc.edu)
}

Received 4 November 2014; Accepted 29 June 2015 cells lost by tissue damage. Research from several laboratories in the last decade has elucidated some of the mechanisms controlling CP. One important mechanism is the induction of proliferation by apoptotic cells. Caspases are required for robust regeneration in many organisms even though caspase activity and cell death contributes to initial tissue loss following damage. When apoptosis is blocked by the baculovirus effector-caspase inhibitor p35 in damaged Drosophila wing discs, 'undead cells', which have initiated but not completed apoptosis, induce hyperproliferation (Huh et al., 2004; Perez-Garijo et al., 2004). This hyperproliferation is dependent on the initiator caspase Dronc, suggesting that this caspase has a role in inducing proliferation independently of apoptosis (Huh et al., 2004). Proliferation induced by undead cells or genuine apoptotic cells may act in various contexts through multiple pathways, including the Wingless/Wnt, Dpp/BMP, Jun N-terminal kinase (JNK), and Hedgehog signaling pathways (Ryoo et al., 2004; Perez-Garijo et al., 2005; Fan and Bergmann, 2008). Apoptosis-induced proliferation involving mitogenic signaling is likely to be just one part of a larger pathway controlling $\mathrm{CP}$ (Mollereau et al., 2013).

The participation of apoptotic cells in regeneration is not unique to Drosophila. Studies in other organisms have revealed that caspase activity is required for regeneration in the Xenopus laevis tadpole tail (Tseng et al., 2007) and in the mammalian liver and skin (Li et al., 2010). Additionally, many of the pathways identified as being involved in CP regulation in Drosophila are also involved in vertebrate tissue repair. For example, both the Hedgehog (Cai et al., 2011) and JNK (Wuestefeld et al., 2013) pathways are required for mammalian liver regeneration, and Wnt signaling is required for limb regeneration in Xenopus, axolotl and zebrafish (Kawakami et al., 2006). Thus, there are conserved mechanisms for regeneration between invertebrates and vertebrates.

How the cell cycle is regulated during regeneration to ensure proper regrowth remains unclear. Tissue regrowth can be accomplished by increasing proliferation to restore cell number, as in Drosophila wing discs, or by cellular growth without cell division, such as during endoreplication (Fox and Duronio, 2013). A strong proliferative response to damage occurs during planaria body regeneration (Wenemoser and Reddien, 2010), zebrafish heart regeneration (Poss et al., 2002) and Xenopus tail regeneration (Tseng et al., 2007), and endoreplication contributes to tissue repair in the mammalian liver (Sigal et al., 1999) and the Drosophila ovary (Tamori and Deng, 2013). In these tissues, a moderate increase in proliferating or endoreplicating cells quickly replaces lost tissue.

By contrast, how cell cycle exit in quiescent tissues is overcome to allow proliferation following damage is unclear. Robust inhibition of cell cycle re-entry in quiescent tissues is necessary to maintain tissue homeostasis and prevent neoplasia and cancer. Cell cycle inhibition thus presents a high hurdle to overcome before regeneration can take place. We investigated this issue using the Drosophila eye imaginal disc. The eye disc contains a 
population of cells that are normally quiescent but will undergo cell cycle re-entry after induction of massive cell death (Fan and Bergmann, 2008). We used the developing eye as a model for CP and performed a genetic screen to identify regulators of this process. With this approach, we identified the transcription factor Scalloped (Sd) as a novel CP regulator. We show that $\mathrm{Sd}$ and the Sd binding partner Yorkie (Yki) are required for cell cycle re-entry following damage in the eye imaginal disc. Yki is a transcriptional effector of the Hippo pathway and was previously identified as a CP regulator in wing discs (Sun and Irvine, 2011; Grusche et al., 2011). We also found that $\mathrm{CP}$ in the eye disc requires the Hippo pathway regulator Ajuba (Jub), similar to recent results in the wing disc (Sun and Irvine, 2013). However, Jub activation during CP is likely to be differentially regulated in these two tissues. Our study demonstrates that Hippo signaling is required for quiescent cells to re-enter the cell cycle following tissue damage and is likely to provide insight into a variety of regenerative systems, particularly those within non-proliferative tissues.

\section{RESULTS}

\section{Quiescent cells re-enter the cell cycle after tissue damage in the developing eye}

The developing Drosophila eye is ideal for studying regeneration in a quiescent cell population. Many genetic tools are available for manipulating the eye imaginal disc, and subtle defects in eye development are readily apparent in the highly organized adult eye
(Dominguez and Casares, 2005; Gutierrez-Avino et al., 2009). The neurocrystalline lattice of the Drosophila eye takes shape by precise control of the cell cycle and differentiation during development (Fig. 1A,L). In early larval development, the eye disc grows as undifferentiated cells proliferate asynchronously. During the third and last larval stage, a wave of differentiation associated with an apical constriction of the epithelial sheet called the morphogenetic furrow (MF) moves from posterior to anterior across the disc. Cells within the MF arrest in G1, and a subset begin to differentiate into photoreceptors. After the MF has passed, the remaining undifferentiated cells synchronously enter $\mathrm{S}$ phase in what is termed the second mitotic wave (SMW) (Fig. 1B). After the SMW, these cells become quiescent and await cues to differentiate (Fig. 1L) (Firth and Baker, 2005). This population is considered to be quiescent as very few cells posterior to the SMW enter S phase (Fig. 1B') or undergo mitosis (Baker and Yu, 2001). Cells remain quiescent by mechanisms that prevent cell cycle re-entry, including CDK inhibition by Dacapo and E2f1 repression by Retinoblastomafamily protein (Rbf) (Buttitta et al., 2007; Ruggiero et al., 2012), as well as destruction of cell cycle regulators by the anaphasepromoting complex (Buttitta et al., 2010; Bandura et al., 2013).

Although cells posterior to the SMW are normally quiescent, cell cycle re-entry occurs after tissue damage (Fan and Bergmann, 2008). Expression of the pro-apoptotic gene hid with the GMR promoter, which is expressed posterior to the MF (supplementary material Fig. S1A), induces extensive cell death (Fig. 1E,F). Dying,
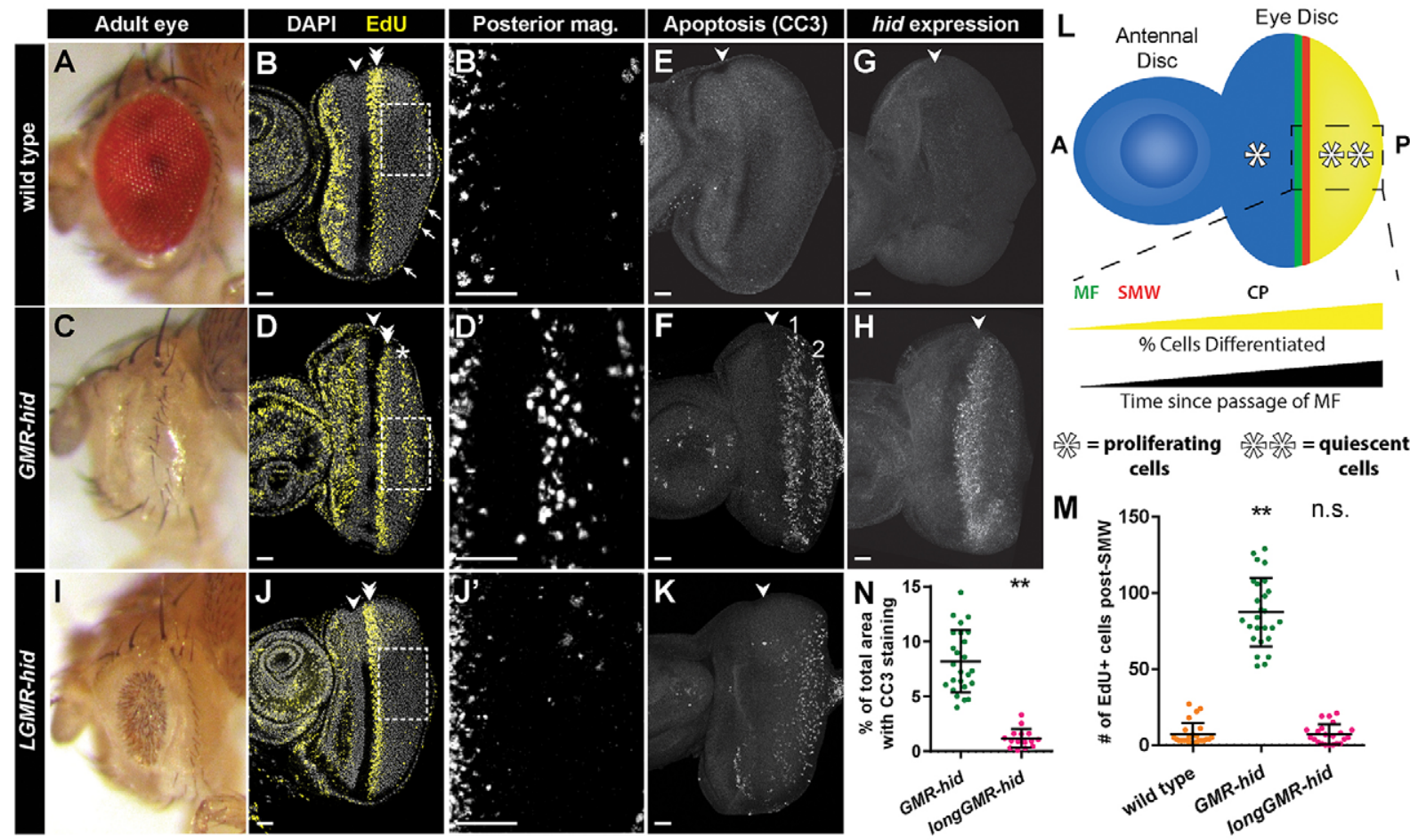

Fig. 1. Hid expression induces $C P$ in the eye imaginal disc. (A,C,I) Adult eyes of the indicated genotypes. Wild-type (A) is Oregon R. (B,D,J) DAPI (DNA; single confocal slice in gray) and EdU (S phase; projection in yellow) staining of eye imaginal discs, indicating the MF (arrowheads) and the SMW (double arrowheads), respectively. Note that $\mathrm{EdU}^{+}$cells in the row of cells around the disc (arrows in $\mathrm{B}$ ) are margin cells and not part of the disc proper. Asterisk denotes $\mathrm{CP}(\mathrm{D})$ ). Boxes indicate areas of magnification shown in $B^{\prime} D^{\prime}, J^{\prime}$. (E,F,K) Cleaved Caspase-3 (CC3) staining of apoptotic cells in discs of the indicated genotypes. The first (1) and second (2) apoptotic waves are indicated in (F). (G,H) In situ hybridization for hid mRNA in the indicated genotypes. (L) The eye disc, attached to the anterior (A) antennal disc, is composed of undifferentiated, proliferating cells (blue) anterior to the MF (green) and both undifferentiated and differentiated quiescent cells (yellow) posterior $(P)$ to the SMW (red). Cells furthest from the MF (most posterior) are the most differentiated. (M) Quantification of CP in the indicated genotypes. All post-SMW, EdU ${ }^{+}$eye disc cells were counted. Each circle represents the number of cells counted for a single disc, and bars represent mean and one standard deviation. For each genotype, $n \geq 22$ discs. ${ }^{* *} P=2.6 \times 10^{-17}$. (N) Quantification of percentage of total disc area with $\mathrm{CC} 3$ staining. Each circle represents the percentage for a single disc, and bars represent mean and one standard deviation. $n>15$ discs. ${ }^{* *} P=2.4 \times 10^{-14}$. n.s., not significant. Anterior is oriented to the left. Scale bars: $20 \mu \mathrm{m}$. 
caspase-positive cells with pyknotic nuclei are extruded from the basal surface of the eye disc (supplementary material Fig. S1B,C), as in other discs (Gibson and Perrimon, 2005; Shen and Dahmann, 2005). Not all cells posterior to the SMW die, and some overcome cell cycle inhibition and re-enter $\mathrm{S}$ phase in a wave of proliferation (Fig. 1D,D'; Fan and Bergmann, 2008). GMR-hid flies are nearly eyeless (Fig. 1C), indicating that increased proliferation cannot fully compensate for tissue loss, probably because GMR-hid expression during pupal stages induces extensive apoptosis after the potential to re-enter the cell cycle is lost. Thus, GMR-hid eye discs behave somewhat differently compared with previous $\mathrm{CP}$ models in the wing where tissue regrowth is more complete (Mollereau et al., 2013). Nonetheless, tissue damage-induced cell cycle re-entry in the eye disc provides a valuable model for studying $\mathrm{CP}$ in a quiescent cell population.

Because apoptotic cells play a crucial role during $\mathrm{CP}$, we further characterized the relationship between dying and proliferating cells in the eye disc. Rather than uniform apoptosis across GMR-hid discs, two distinct waves of cleaved Caspase-3 (CC3)-positive cells occur on either side of the $\mathrm{CP}$ wave (Fig. 1E,F; supplementary material Fig. S1D-E). Because CP overlaps substantially with the apoptosis-free zone between the two waves of CC3 staining, it was previously suggested that proliferating cells might inhibit apoptosis (Fan and Bergmann, 2008). However, these two waves of apoptosis persist when $\mathrm{CP}$ is blocked (see below and Fig. 3B,C), suggesting that $\mathrm{CP}$ does not inhibit apoptosis. Rather, variations in Hid activity across the disc may account for the observed pattern of apoptosis. Hid accumulation and activity varies posterior to the MF (Fan and Bergmann, 2014). In addition, hid mRNA is not uniformly expressed across GMR-hid discs, with high levels immediately posterior to the MF and lower levels more posteriorly (Fig. 1G,H). hid mRNA can be regulated by the miRNA bantam (ban) (Brennecke et al., 2003), which protects cells from apoptosis in certain damaged tissues after being upregulated by a Tie-like receptor tyrosine kinase (Tie)-dependent mechanism (Bilak et al., 2014). We did not detect ban induction in GMR-hid discs (supplementary material Fig. S5C), and Tie RNAi does not affect the pattern of apoptosis (supplementary material Fig. S2A). These results suggest that Tie-dependent ban induction is not responsible for decreased hid transcripts in the posterior eye disc.

Because hid transcripts are low in the posterior of the disc, we considered the possibility that dying cells in the first apoptotic wave promote apoptosis-induced apoptosis (AiA), resulting in the second apoptotic wave. AiA is mediated by JNK signaling (Perez-Garijo et al., 2013). However, expression of a dominantnegative version of the Drosophila JNK homolog Basket (Bsk) does not affect the pattern of apoptosis in GMR-hid discs (supplementary material Fig. S2B). Interestingly, although JNK signaling is required for CP in the wing disc (Ryoo et al., 2004), expression of $\mathrm{Bsk}^{\mathrm{DN}}$ or Puckered (Puc), a negative regulator and downstream target of the JNK pathway, does not suppress CP (supplementary material Fig. S2C,D,G). Additionally, although $p u c$ is induced after damage in the wing (Bergantinos et al., 2010), puc-lacZ is not induced in GMR-hid eye discs (supplementary material Fig. S2E,F). We conclude from these data that regulation of Hid expression and activity, rather than S-phase entry, ban induction, or AiA, determines the pattern of apoptosis in GMRhid discs and that JNK signaling is not a major CP regulator posterior to the MF.

Next, we explored contributions to the pattern of CP in GMR-hid discs. CP occurs in a well-defined wave that does not typically extend to the posterior edge of the disc (Fig. 1D, $\mathrm{D}^{\prime}$ ). Undifferentiated cells, which are the only cells that re-enter S phase in GMR-hid discs (Fan and Bergmann, 2008), are present at the posterior edge of the eye disc. We hypothesized that cells lose the competency to re-enter S phase prior to differentiation, perhaps owing to prolonged quiescence. To address this hypothesis, we shifted the wave of apoptosis towards the posterior edge of the disc by expressing hid under the control of longGMR ( $L G M R)$, a version of the GMR promoter that contains a repressor element and is only expressed in a subset of photoreceptors (Wernet et al., 2003; supplementary material Fig. S1F). Although we were unable to obtain clear hid mRNA signal in this genotype, probably owing to lower levels of accumulation than in GMR-hid discs, we did observe a single wave of apoptosis in the posterior region of $L G M R$-hid discs (Fig. 1K). In addition, LGMR flies have a reduced adult eye phenotype similar to, but less severe than, GMR-hid flies (Fig. 1I). Unlike in GMR-hid discs, however, CP is not induced in LGMR-hid discs (Fig. $1 \mathrm{~J}, \mathrm{~J}^{\prime}, \mathrm{M}$ ). This result is consistent with the idea that undifferentiated cells at the posterior of the disc are refractory to cell cycle re-entry in response to tissue damage. Alternatively, lower levels of apoptosis in LGMR-hid discs compared with GMR-hid discs (Fig. 1N) may not be sufficient to induce CP.

\section{An RNAi screen identifies genes required for compensatory proliferation}

How do undifferentiated cells overcome quiescence to re-enter the cell cycle in response to tissue damage? The mechanisms that control $\mathrm{CP}$ in quiescent eye discs are distinct from those in proliferating wing discs (Fan and Bergmann, 2008). For example, JNK signaling is required for CP in the wing disc (Ryoo et al., 2004) whereas our data suggest it is not required in the eye disc (supplementary material Fig. S2). To identify pathways necessary for cell cycle re-entry in the regenerating eye, we designed a genetic screen based on adult eye phenotypes. We expressed hairpin RNAs targeting individual genes in GMR-hid eye discs using the GAL4/ UAS system and assessed the effect on CP by scoring the adult eye phenotype. To drive UAS-transgene expression, we constructed a GMR-hid, GMR-Gal4/+ (GMR>hid, Gal4) line (unless otherwise noted, genotypes written as GMR>hid, transgene are heterozygous for GMR-hid, GMR-Gal4, and a UAS-transgene). Eyes in GMR $>$ hid, Gal4 flies are drastically reduced in size compared with wild type but larger than GMR-hid eyes (compare Fig. 1A,C and Fig. 2A). GMR>hid, Gal4 larval eye discs exhibit strong CP (Fig. 2A') with similar numbers of cells re-entering $\mathrm{S}$ phase compared with $G M R$-hid $(P=0.39)$. We tested whether inhibiting $\mathrm{CP}$ would result in a detectable adult eye phenotype by expressing the mammalian CDK inhibitor p21 or dsRNA targeting Cyclin E in GMR > hid, Gal4 eye discs. In these genotypes, CP is blocked and a reproducible adult eye phenotype results in which pigment is reduced (Fig. 2B-C'). Because pigment cells are one of the last cell types to differentiate, this result suggests that the undifferentiated, precursor pool is reduced when CP is blocked and that cells that undergo CP contribute to the adult eye. Importantly, the SMW does not appear to be affected in GMR-Gal4, GMR-hid/UAS-p21 discs (supplementary material Fig. S3A,B), in contrast to GMR-p21 flies in which the SMW is ablated (supplementary material Fig. S3C; de Nooij and Hariharan, 1995). These data suggest that the accumulation of Gal4 required to drive high UAS-transgene expression does not occur until posterior to the SMW, allowing us to assess phenotypes during $\mathrm{CP}$ without confounding defects in the SMW. In addition, these data indicate that we can detect loss of $\mathrm{CP}$ by monitoring adult eye phenotypes. 
Using this approach, we screened a collection of UAS-RNAi lines targeting Drosophila transcription factors. Our rationale for this strategy is that many signaling pathways affecting cell cycle entry and CP have transcriptional output. In addition, multiple inputs can result in activation of the same transcription factor, and we reasoned that knocking down the transcription factor itself would result in a stronger phenotype than knocking down an upstream component. Of the 544 transcription factors included in both the Fly Transcription Factor Database (Pfreundt et al., 2010) and the Animal Transcription Factor Database (Zhang et al., 2012), we tested the 373 genes for which there was an available RNAi line (supplementary material Table S1). In our primary screen, candidate genes were identified based on suppression or enhancement of the GMR > hid, Gal4 adult eye phenotype (Fig. 2D). luciferase (luc) RNAi was used as a negative control. Twelve UAS-RNAi lines caused lethality with both GMR>hid, Gal4 and GMR-Gal4 alone and were not examined further (supplementary material Table S1).

Fifty two UAS-RNAi lines modified the GMR $>$ hid, Gal4 adult eye phenotype: three acted as suppressors, including glass, which binds to and activates GMR (Fig. 2E; supplementary material Fig. S4); twelve displayed a small eye (Enhancer Category I; Fig. 2F; supplementary material Fig. S4); seven displayed moderate pigment loss (Enhancer Category II; Fig. 2G; supplementary material Fig. S4); twenty one displayed mild pigment loss (Enhancer Category III; Fig. 2H; supplementary material Fig. S4); and two fell into a category we termed 'Other' (supplementary material Fig. S4). These 52 lines were also crossed to GMR-Gal4 alone to determine whether they disrupted eye development independently of GMR-hid (supplementary material Fig. S4). Because many UAS-RNAi lines caused a rough eye phenotype with GMR-Gal4 alone and thus may disrupt eye development in a process distinct from $\mathrm{CP}$, we performed a secondary screen to assess $\mathrm{CP}$ within the larval eye discs (Fig. 2D). In many of the $52 \mathrm{RNAi}$ lines tested, we observed normal or slightly disrupted $\mathrm{CP}$ (supplementary material Fig. S4). Four RNAi lines caused a striking loss of CP: Dp, scalloped ( $s d$ ), fork head and knirps. Dp is known to be required for entry into $\mathrm{S}$ phase (Frolov et al., 2005) and thus was not characterized further. Of the remaining three genes, we focused our studies on $\mathrm{Sd}$, a TEAD/TEF transcription factor that regulates growth and apoptosis in many developing tissues (Simmonds et al., 1998; Goulev et al., 2008; Wu et al., 2008; Zhang et al., 2008).

\section{Scalloped is required for compensatory proliferation}

$\mathrm{CP}$ is substantially reduced in GMR-hid discs by sd knockdown with either of two independently derived RNAi lines (Fig. 3A,B,G). To verify this result, we tested whether a $s d$ mutation would also block CP. Because sd null mutants are embryonic lethal (Deshpande et al., 1997), we generated $s d$ mutant clones in GMRhid eye discs using the ey>FLP/FRT system (Fig. 3C-C'"). Whereas wild-type clones contain EdU-positive cells in the position of the $\mathrm{CP}$ wave, sd mutant clones do not (Fig. 3C", $\mathrm{C}^{\prime \prime \prime}$ ). Although apoptosis is slightly decreased in GMR>hid, sd RNAi discs compared with GMR $>$ hid, luc RNAi (Fig. 3A,B,H), the decrease in $\mathrm{CP}$ is not due to the decrease in apoptosis because many $G M R>h i d$, sd RNAi discs have the same amount of apoptosis as controls, whereas none has the same degree of $\mathrm{CP}$ as controls (Fig. $3 \mathrm{H}$ ). Taken together, these results indicate that $\mathrm{Sd}$ is a $\mathrm{CP}$ regulator.

Recent results suggest that in certain contexts $\mathrm{Sd}$ can act as a suppressor of genes controlling apoptosis and growth. This

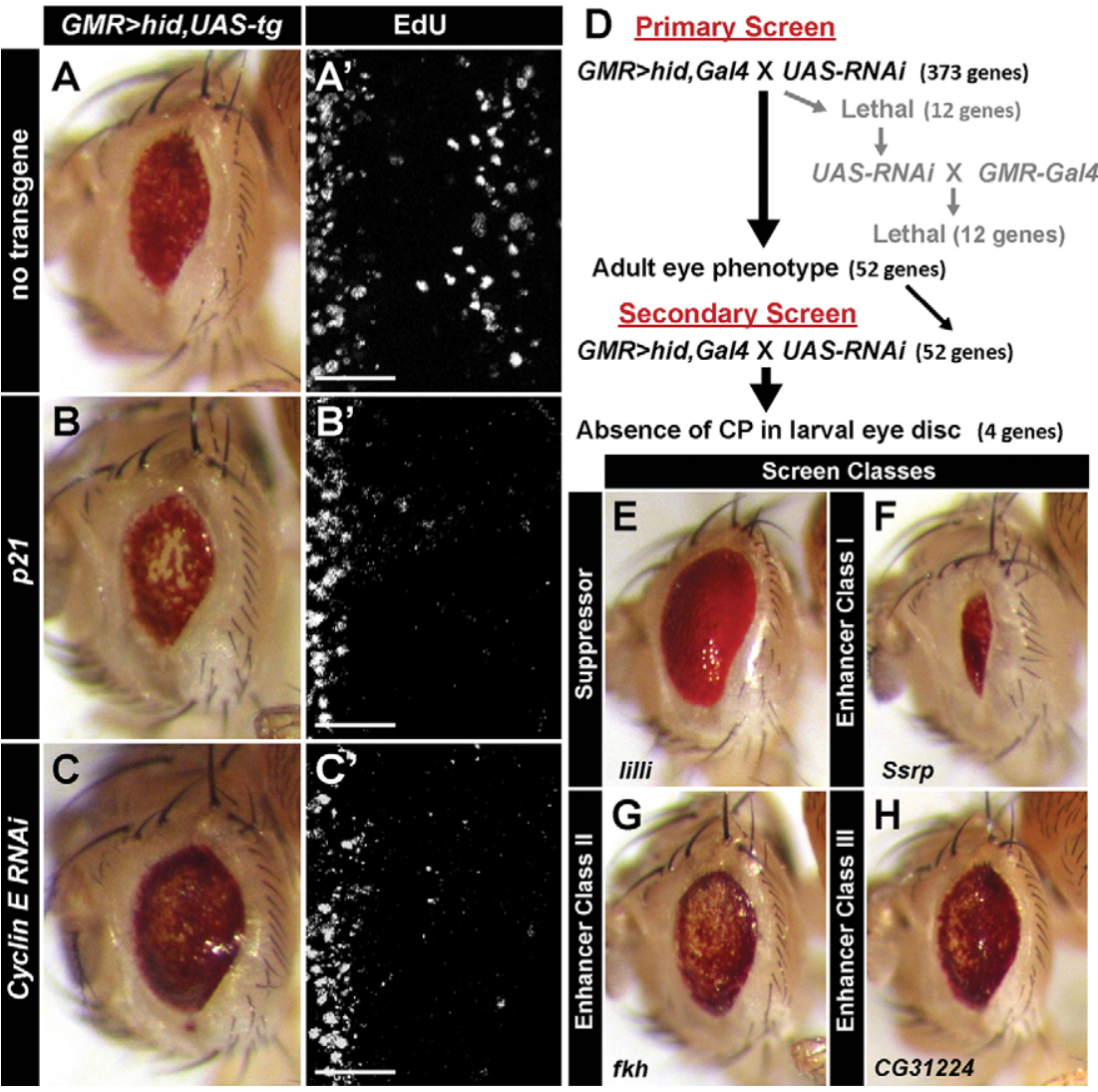

Fig. 2. Genetic screen for regulators of compensatory proliferation. (A-C') Adult eyes (A-C) and high magnification view of posterior eye imaginal discs $\left(A^{\prime}-C^{\prime}\right.$; as in Fig. 1 $\mathrm{B}^{\prime}$ ) expressing the indicated UAS-transgenes in GMR-hid, GMR-Gal4/+ individuals. (D) Schematic of the RNAi screen. (E-H) Representative examples of the four categories of adult eye phenotypes resulting from the RNAi screen. See also supplementary material Table $\mathrm{S} 1$ and Figs S3,S4. Anterior is oriented to the left. Scale bars: $20 \mu \mathrm{m}$. 

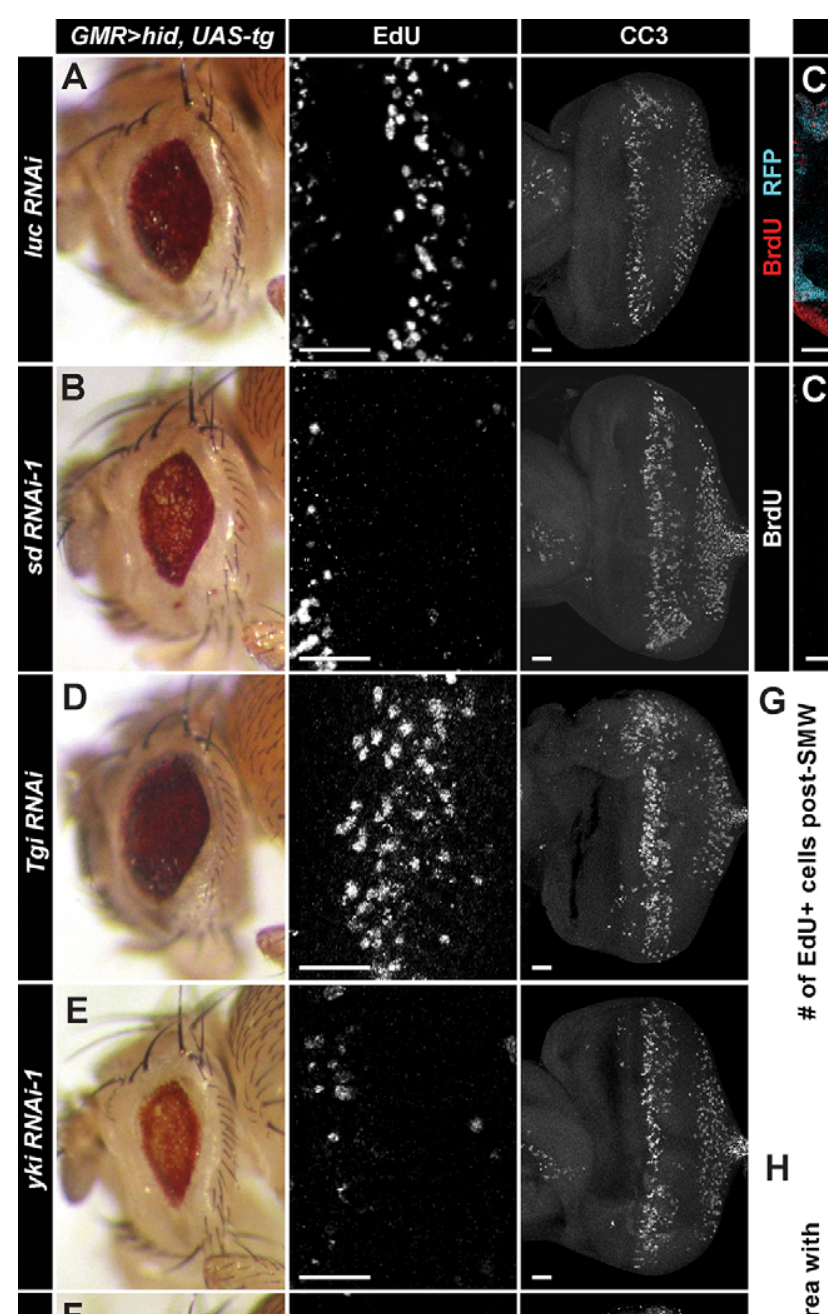

FRT sd / FRT RFP; GMR-hid / ey>FLP
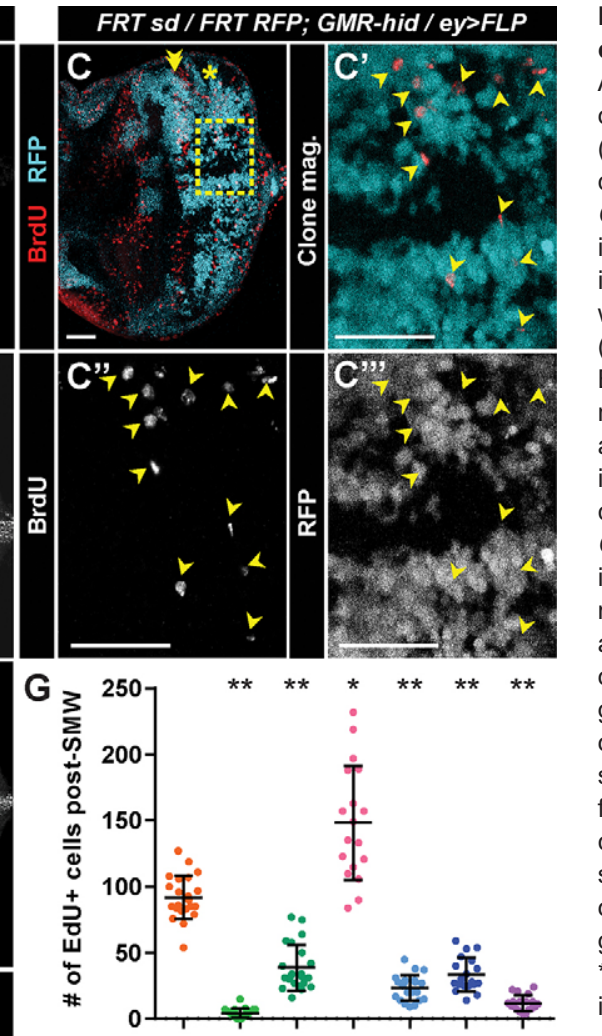

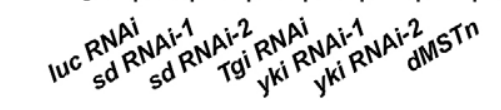

$\mathrm{H}$
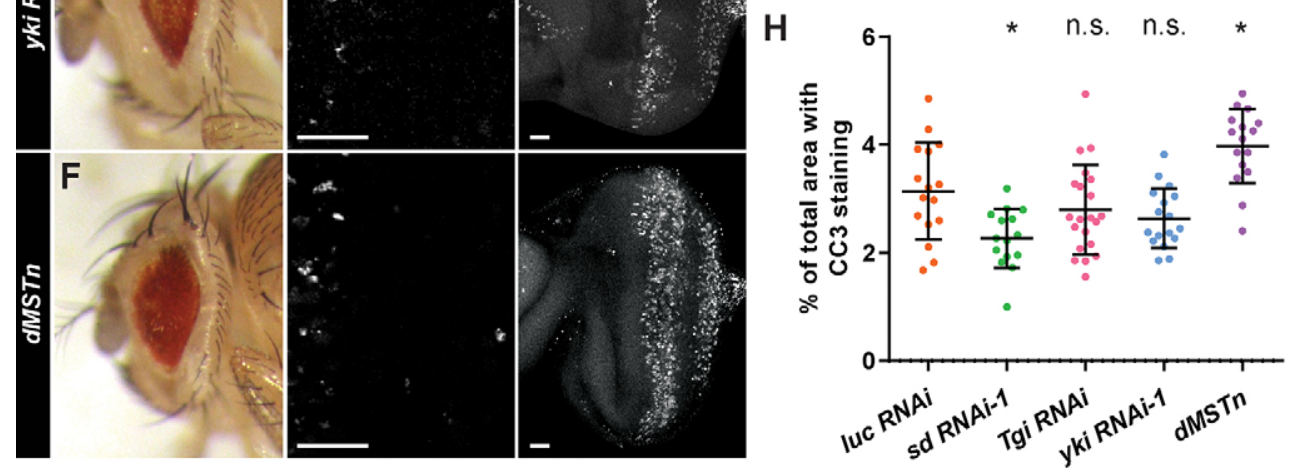

Fig. 3. sd and yki are required for compensatory proliferation. (A,B,D-F)

Adult eyes (left panels), high magnification of posterior eye discs stained with EdU (middle panels), and apoptosis as detected by CC3 staining (right panels) in GMR>hid, Gal4 discs expressing the indicated transgenes. luciferase (IUc) RNAi is used as a control (A). (C-C'") Clones of wild-type (RFP ${ }^{+}$, cyan) and $s d$ mutant $\left(\mathrm{RFP}^{-}\right)$cells in the GMR-hid background. Boxed area in $\mathrm{C}$ indicates area of magnification shown in $\mathrm{C}^{\prime}-\mathrm{C}^{\prime \prime \prime}$. Double arrowhead indicates SMW; asterisk indicates CP. Arrowheads indicate $\mathrm{BrdU}^{+}$ cells. (G) Quantification of CP in the GMR>hid, Gal4 background for the indicated UAS-transgenes. Each circle represents the number of cells counted for a single disc, and bars represent mean and one standard deviation. For each genotype, $n \geq 19$ discs. (H) Quantification of percentage of total disc area with CC3 staining in the GMR>hid, Gal4 background for the indicated UAS-transgenes. Each circle represents the percentage for a single disc, and bars represent mean and one standard deviation. For each genotype, $n \geq 15$ discs. ${ }^{*} P \leq 3 \times 10^{-3}$,

${ }^{* *} P \leq 3 \times 10^{-13}$. n.s., not significant. Anterior is oriented to the left. Scale bars: $20 \mu \mathrm{m}$. suppressor activity is dependent on the Tondu-domain-containing Growth Inhibitor (Tgi) co-factor (Koontz et al., 2013). We reasoned that the slight decrease in apoptosis in GMR>hid, sd RNAi discs could reflect $\mathrm{Sd}$ suppressor activity. However, the amount of apoptosis is unchanged in GMR>hid, Tgi RNAi discs relative to control (Fig. 3D,H). Interestingly, CP increases in GMR>hid, Tgi $R N A i$ discs (Fig. 3D,G), possibly as a result of increased growth gene expression (Fig. 3D). The opposing phenotypes of $s d$ and Tgi RNAi in the GMR-hid background make it unlikely that Sd is acting as a suppressor to induce CP.

\section{Yorkie is required for compensatory proliferation}

Sd activates expression of target genes in the eye disc as part of a complex with Yorkie (Yki), the transcriptional effector of the Hippo pathway (Goulev et al., 2008; Wu et al., 2008; Zhang et al., 2008). The Hippo pathway controls tissue growth by regulation of Yki, targets of which include cell cycle regulators, such as Cyclin E (Huang et al., 2005) and E2f1 (Goulev et al., 2008), and anti- apoptotic genes, such as Diapl (Huang et al., 2005) and ban (Thompson and Cohen, 2006). Flux through the Hippo pathway is governed by Hippo (Hpo) phosphorylation of Warts (Wts), which in turn phosphorylates Yki. Phosphorylated Yki cannot translocate to the nucleus (Oh and Irvine, 2008). Thus, increased Hippo signaling reduces transcription of Yki target genes. Unphosphorylated, nuclear Yki acts as a co-activator for transcription factors, such as Sd and the Homothorax (Hth)/Teashirt (Tsh) complex (Peng et al., 2009). Because Yki itself does not bind DNA, it was not included in our initial screen. Targeting $y k i$ with two independently derived UAS-RNAi transgenes reduced CP and resulted in an adult eye phenotype similar to $s d$ RNAi (Fig. 3E,G). Levels of apoptosis in $G M R>h i d, y k i R N A i$ discs were not significantly different from controls (Fig. $3 \mathrm{H}$ ). We further probed the contribution of Yki to CP by overexpressing the Hpo kinase domain (dMSTn; Zhang et al., 2008), which blocks Yki activity. The adult eye phenotypes were similar to those elicited by $s d$ or $y k i$ RNAi (Fig. 3F), and CP in the eye disc was significantly reduced compared with controls 
(Fig. 3F,G). We conclude that Yki is necessary to induce CP in eye discs, suggesting a shared role for Hippo signaling in regulating $\mathrm{CP}$ in eye and wing discs.

\section{Cyclin E is induced by Sd/Yki during compensatory proliferation}

$\mathrm{Sd} /$ Yki targets include Cyclin E, Diap1, expanded (ex) and ban (Wu et al., 2008; Zhang et al., 2008). As Cyclin E is required for CP (Fig. 2C), we compared Cyclin E levels in wild-type and GMR-hid discs. In proliferating cells, like those anterior to the MF or within the SMW, Cyclin E levels rise during G1 to induce entry into S phase (Fig. 4A) (Knoblich et al., 1994), then fall during S phase and mitosis as a result of transcriptional (Duronio and O'Farrell, 1995) and post-translational (Moberg et al., 2001) regulation. In wild-type eye discs, Cyclin E levels are low in quiescent cells posterior to the SMW (Fig. 4B) (Richardson et al., 1995). By contrast, Cyclin E expression is high posterior to the SMW in GMR-hid and GMR>hid, luc RNAi discs (Fig. 4C,D). This increase in Cyclin E accumulation occurred primarily in $\mathrm{Yan}^{+}$ undifferentiated cells rather than in photoreceptors (Fig. 4C,D). $s d$ or $y k i$ RNAi reduces Cyclin E accumulation posterior to the SMW (Fig. 4E-G). These data suggest that $\mathrm{Sd} / \mathrm{Yki}$ induce Cyclin $\mathrm{E}$
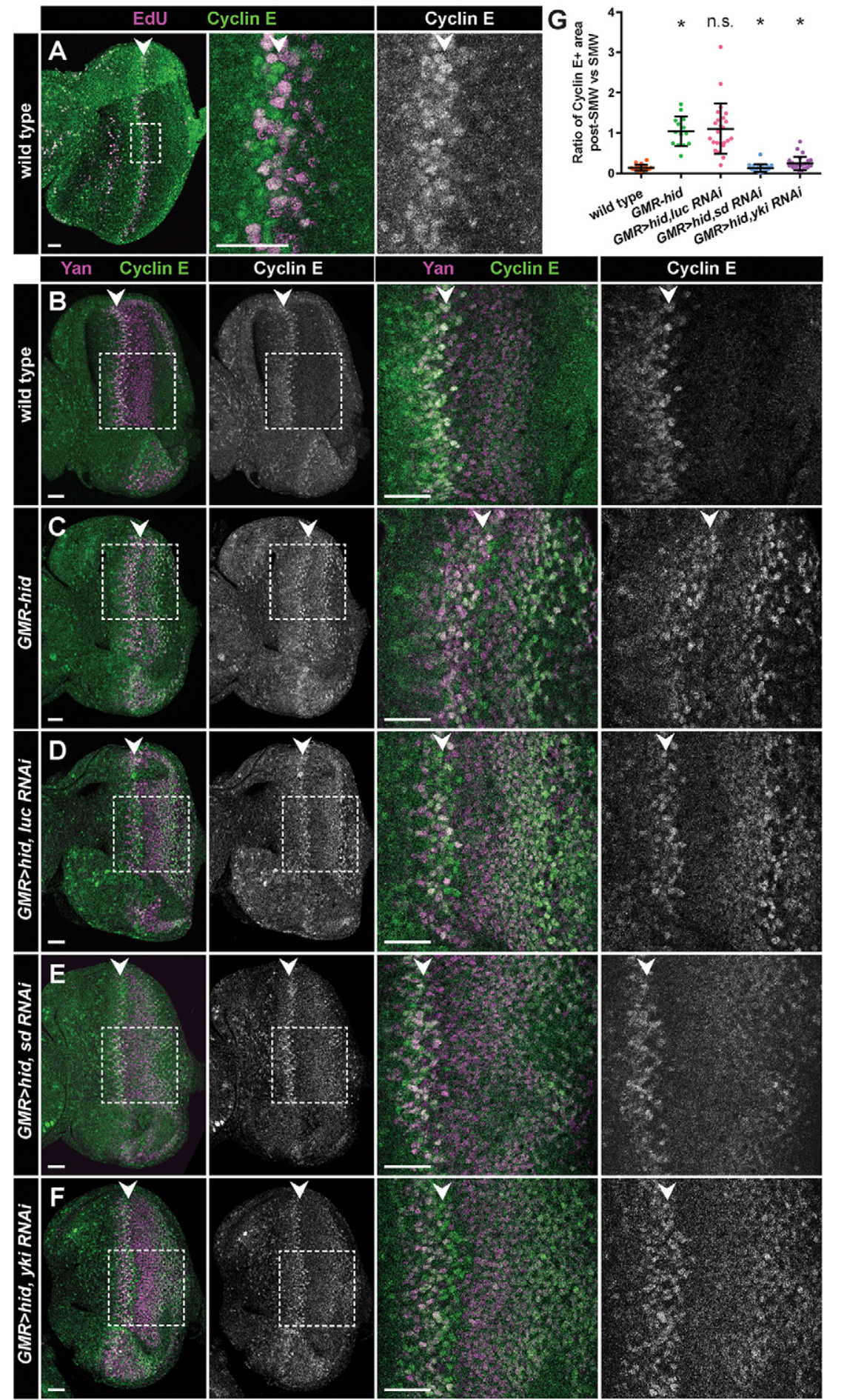

Fig. 4. Sd and Yki are required for elevated

Cyclin E levels during CP. (A) Eye disc labeled with EdU (magenta) and anti-Cyclin E (green) antibodies. Cyclin E accumulates prior to and during $S$ phase of the SMW (arrowhead). Box in left panel indicates area of magnification in middle and right panels. (B-F) Eye discs of the indicated genotypes stained with anti-Cyclin $\mathrm{E}$ (green) and anti-Yan (marker of undifferentiated cells; magenta) antibodies. Boxes indicate areas shown at higher magnification on the right. Arrowheads indicate SMW.

(G) Quantification of Cyclin E staining in $\mathrm{Yan}^{+}$cells of the indicated genotypes. The ratio of post-SMW Cyclin E staining versus SMW Cyclin E staining is displayed (see supplementary Methods for details). Each circle represents the ratio calculated for a single disc, and bars represent mean and one standard deviation. For each genotype, $n \geq 14$ discs. ${ }^{*} P \leq 1.8 \times 10^{-7}$. Significance was calculated for wild type $\left(w^{1118}\right)$ versus GMR-hid, GMR-hid versus $G M R>h i d$, luc $R N A i$, GMR>hid, luc RNAi versus GMR>hid, sd RNAi, and GMR>hid, luc RNAi versus $G M R>h i d, y k i$ RNAi. n.s., not significant. Anterior is oriented to the left. Scale bars: $20 \mu \mathrm{m}$. 
expression in regenerating eye discs, driving S-phase entry during $\mathrm{CP}$.

We next examined expression of the Yki targets ex and ban in GMR-hid tissues. Whereas we observe CP (supplementary material Fig. S5A) and apoptosis (supplementary material Fig. S5B) in GMR-hid clones, we do not observe differences in expression of a transgene that detects ban activity (Brennecke et al., 2003) (supplementary material Fig. S5C) or ex-lacZ expression (supplementary material Fig. S5D) between wild-type and GMRhid clones. Additionally, we do not observe an increase in ban sensor expression or ex-lacZ in GMR-hid discs compared with wildtype discs (supplementary material Fig. S5E,F).

\section{Yki overexpression rescues GMR-hid phenotypes}

Because Yki overexpression induces Cyclin $E$ expression and proliferation (Huang et al., 2005), we tested whether increased Yki expression rescues GMR-hid phenotypes. We utilized both wildtype Yki and a version of Yki ( $\mathrm{Yki}^{\mathrm{S}}{ }^{168 \mathrm{~A}}$ ) that is hyperactive owing to reduced phosphorylation by Wts (Oh and Irvine, 2008). GMRGal4-driven expression of either Yki or $\mathrm{Yki}^{\mathrm{S} 168 \mathrm{~A}}$ induces Cyclin $\mathrm{E}$ and ectopic S-phase entry, but not apoptosis, posterior to the MF (supplementary material Fig. S6). Yki ${ }^{\mathrm{S} 168 \mathrm{~A}}$ expression induces a considerable number of cells to enter $\mathrm{S}$ phase, resulting in extensive overgrowth in both the larval disc (supplementary material Fig. S6B") and the adult eye (supplementary material Fig. S6A"). Similar results were obtained in the GMR>hid, Gal4 background (Fig. 5). Both Yki and $\mathrm{Yki}^{\mathrm{S} 168 \mathrm{~A}}$ expression result in high Cyclin $\mathrm{E}$ accumulation throughout the posterior of GMR $>$ hid, Gal4 discs (Fig. 5C-C"). Although the adult eye morphology, CP and apoptosis appear similar in control and GMR>hid, yki discs (Fig. $5 \mathrm{~A}^{\prime}, \mathrm{B}^{\prime}, \mathrm{D}^{\prime}$ ), there is an increase of cells in $\mathrm{S}$ phase in $G M R>h i d, y k i^{S 168 A}$ discs (Fig. 5B"), and overgrowth occurs in both the disc and the adult eye (Fig. 5A", $\mathrm{B}^{\prime \prime}$ ). Therefore, overexpression of $\mathrm{Yki}^{\mathrm{S} 168 \mathrm{~A}}$ rescues the small eye phenotype caused by GMR-hid, indicating that Yki activation is sufficient to overcome cell loss after Hid induction.

Although suppression of the GMR $>$ hid, Gal4 eye phenotype by $\mathrm{Yki}^{\mathrm{S} 168 \mathrm{~A}}$ expression is likely to be due primarily to extensive overproliferation, we also measured apoptosis in this genotype. Apoptosis is significantly decreased in GMR $>h i d, y k i i^{S 168 A}$ discs, particularly in the second apoptotic wave (Fig. 5D",G). As Diapl is a Yki target and suppressor of apoptosis, we measured Diap1-lacZ levels after $\mathrm{Hid}$ and $\mathrm{Yki}^{\mathrm{S} 168 \mathrm{~A}}$ expression. Diap1-lacZ levels posterior to the furrow increase after Hid expression and increase further with Hid and Yki ${ }^{\text {S168A }}$ expression (Fig. 5E,F). Thus, Ykidependent Diap1 expression may play a role in limiting cell death after hid induction. Although apoptosis is not increased in $G M R>h i d$, yki RNAi discs, apoptosis is increased in GMR>hid, $d M S T n$ discs (Fig. 3F,H). GMR>hpo discs also display moderate apoptosis, independently of Hid expression (Verghese et al., 2012). These data suggest that in addition to its role in CP, Yki activity can regulate apoptosis during tissue damage.

Our data suggest that Yki activation is a key step in CP initiation. We therefore hypothesized that Yki activity is limiting for entry into $\mathrm{S}$ phase during CP. If true, an increase in Yki posterior to the MF would sensitize cells to $\mathrm{CP}$ signals, resulting in earlier S-phase entry that would manifest in a shift of the $\mathrm{CP}$ wave towards the anterior. Indeed, the distance between the anterior border of the SMW and the anterior border of the compensatory wave in $G M R>h i d, y k i$ discs is decreased compared with GMR $>$ hid, luc $R N A i$ (Fig. 5B,H). Because distance along the anteroposterior axis of the eye disc is a proxy for time, these data indicate that cells re-enter S phase sooner in GMR-hid discs expressing additional Yki compared with those without. These data suggest that levels of Yki are important for controlling $\mathrm{CP}$ timing and that active Yki may be the limiting factor for inducing cell cycle re-entry.

\section{Ajuba, an inhibitor of Hippo signaling, is required for compensatory proliferation}

How is Hippo signaling inhibited to allow Yki activation during tissue regeneration? Flux through the Hippo pathway is modulated by events at the cell cortex that monitor epithelial integrity and cellcell interaction (Yu and Guan, 2013). One mechanism for inhibiting Hippo signaling is through the LIM domain protein Ajuba (Jub). Jub antagonizes Hippo signaling and is essential for eye development (Das Thakur et al., 2010). Activated Jub is thought to inhibit Hippo signaling by binding Wts, thereby preventing Wts from phosphorylating Yki (Rauskolb et al., 2014). jub RNAi enhances the GMR > hid, Gal4 adult eye phenotype and reduces CP (Fig. 6A,B,L). This observation is consistent with observations in the wing disc where reduced Jub inhibits regeneration (Sun and Irvine, 2013). Jub localizes with DE-cadherin (Shotgun - FlyBase) at apical adherens junctions in larval eye discs (Fig. 6C), as in pupal eye discs (Das Thakur et al., 2010) and wing discs (Sun and Irvine, 2013). This localization is most apparent at the apical surface of photoreceptors (Fig. 6D). We did not observe obvious accumulation or re-localization of Jub in GMR-hid clones (Fig. 6E), suggesting that increased activation rather than re-localization of Jub may be required to inhibit Hippo signaling during $\mathrm{CP}$.

Jub can be activated in wing discs by JNK signaling (Sun and Irvine, 2013) or by an increase in cellular tension (Rauskolb et al., 2014). Because our data suggest that JNK signaling does not play a significant role in $\mathrm{CP}$ in the eye disc (supplementary material Fig. S2B-G), we investigated whether increased cellular tension modulates $\mathrm{CP}$. We induced an increase in cellular tension by expressing the catalytic domain of Rho kinase (RokCAT), which phosphorylates myosin and leads to increases in myosin contractility (Winter et al., 2001). In GMR>hid, RokCAT discs, CP increases by twofold, although the adult eye phenotype is not noticeably altered (Fig. 6F,L). Conversely, Rok RNAi results in a mild but statistically significant decrease in CP (Fig. 6L). Rok is activated by the Rho1 GTPase (Warner et al., 2010), which is inhibited in the pupal retina by $\mathrm{Cdc} 42$ (Warner and Longmore, 2009). Cdc42 also inhibits CP in irradiated wing discs (Warner et al., 2010). We observed an increase in CP in GMR-hid discs with expression of dominant-negative $\mathrm{Cdc} 42\left(\mathrm{Cdc} 42^{\mathrm{DN}}\right)$ (Fig. 6I,L). These results suggest that regulation of cellular tension through $\mathrm{Cdc} 42 / \mathrm{Rho} / \mathrm{Rok}$ plays a role in $\mathrm{CP}$, presumably through Jub regulation.

We predicted that increases in cellular tension in the absence of Hid expression would also activate Jub and induce cell cycle re-entry. Indeed, when RokCAT was expressed with GMR-Gal4 alone, we observed ectopic $\mathrm{S}$ phases in the posterior of the disc (Fig. 6G,L). We also observed a low level of apoptosis in $G M R>$ RokCAT eye discs (Fig. 6G), similar to previous results in wing discs (Warner et al., 2010). We investigated whether the cell cycle entry in the posterior of GMR $>$ RokCAT discs depends on the presence of these apoptotic cells by labeling $G M R>R o k C A T$, p35 eye discs with EdU and anti-CC3 antibodies. p35 blocks RokCAT-induced apoptosis, which we confirmed by the lack of basally extruded cells and pyknotic nuclei, but does not block CC3 staining owing to the presence of non-cleaved-caspase epitopes of the anti-CC3 antibodies in undead cells (Fan and Bergmann, 2010). Suppression of apoptosis in GMR $>$ RokCAT, p35 discs did not prevent the appearance of ectopic EdU-positive 


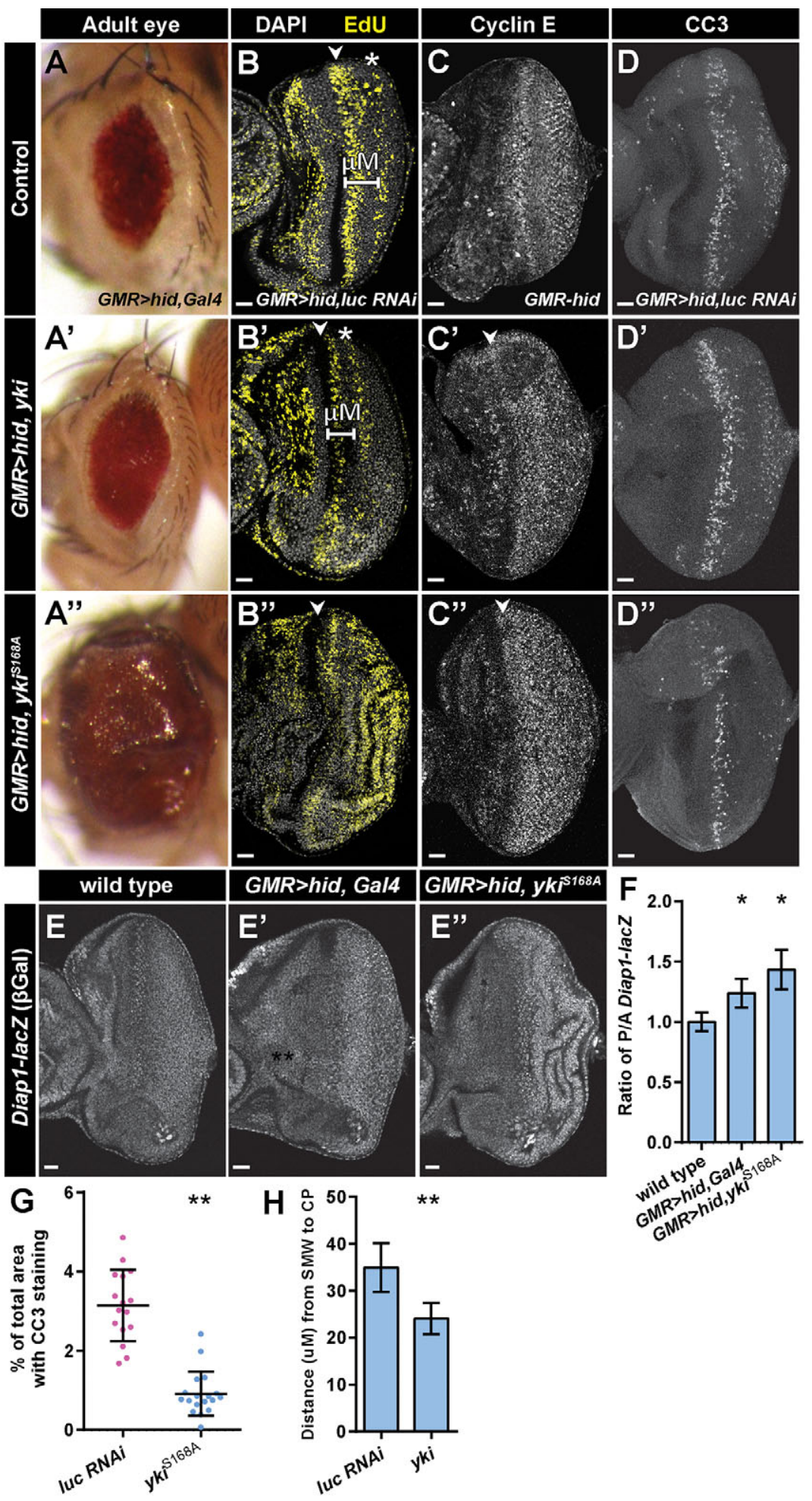

Fig. 5. Expression of transgenic yki modifies the GMR-hid phenotype. (A-A") GMR>hid, Gal4 adult eyes (control in A) after expression of $y k i\left(\mathrm{~A}^{\prime}\right)$ or $y k i^{S 168 A}\left(\mathrm{~A}^{\prime \prime}\right)$. (B-B" $\left.\mathrm{B}^{\prime \prime}\right)$ EdU staining (yellow) in eye discs of the indicated genotypes. Arrowheads indicate SMW. The white bar $\left(B, \mathrm{~B}^{\prime}\right)$ indicates the distance measured between the SMW and CP (asterisk) (data shown in $\mathrm{H}) .\left(\mathrm{C}-\mathrm{C}^{\prime \prime}\right)$ Cyclin E staining of the indicated genotypes. (D-D") CC3 staining of the indicated genotypes. $\left(E-E^{\prime \prime}\right) \beta-G a$ staining of the indicated genotypes detects Diap1-lacZ expression. (F) Quantification of Diap1-lacZ expression by $\beta$-Gal staining [ratio of posterior to anterior (P/A) eye disc, normalized to $w^{1118}$ control] in the indicated genotypes. Error bars represent one standard deviation. ${ }^{*} P \leq 6 \times 10^{-4}, n \geq 11$ discs. (G) Quantification of percentage of total disc area with post-furrow $\mathrm{CC} 3$ staining in GMR>hid, Gal4 eye discs expressing luc RNAi $(n=16)$ or $y k^{S 168 A}(n=18)$. Each circle represents the percentage calculated for a single disc, and bars represent mean and one standard deviation

${ }^{* *} P=3.64 \times 10^{-6}$. (H) Quantification of the distance between the SMW and CP wave in GMR>hid, Gal4 eye discs expressing luc RNAi $(n=25)$ or $y k i$ RNAi $(n=22) .{ }^{* *} P=8.47 \times 10^{-11}$. Error bars represent one standard deviation. Anterior is oriented to the left. Scale bars: $20 \mu \mathrm{m}$

cells posterior to the SMW, indicating that RokCAT directly induces S-phase entry (Fig. 6H,L). We also observed cell cycle re-entry in $G M R>C d c 42^{D N}$ discs, even when p35 was coexpressed (Fig. 6J-L). In wing discs, co-expression of RokCAT and p35 induces hyperproliferation (Warner et al., 2010), which could be attributed to the formation of undead cells rather than increases in tension directly inducing proliferation. However, as p35 expression blocks CP in post-furrow eye discs and does not induce mitogenic undead cells (Fan and Bergmann, 2008), our results suggest that modulation of cellular tension directly induces cell cycle re-entry posterior to the SMW.

\section{DISCUSSION}

To maintain tissue homeostasis and prevent inappropriate cell divisions, the threshold for S-phase entry is higher in quiescent cells than in cycling cells. Consequently, to undergo regeneration efficiently, quiescent tissues must overcome robust controls that restrain cell cycle entry. The mechanisms that drive cell cycle 

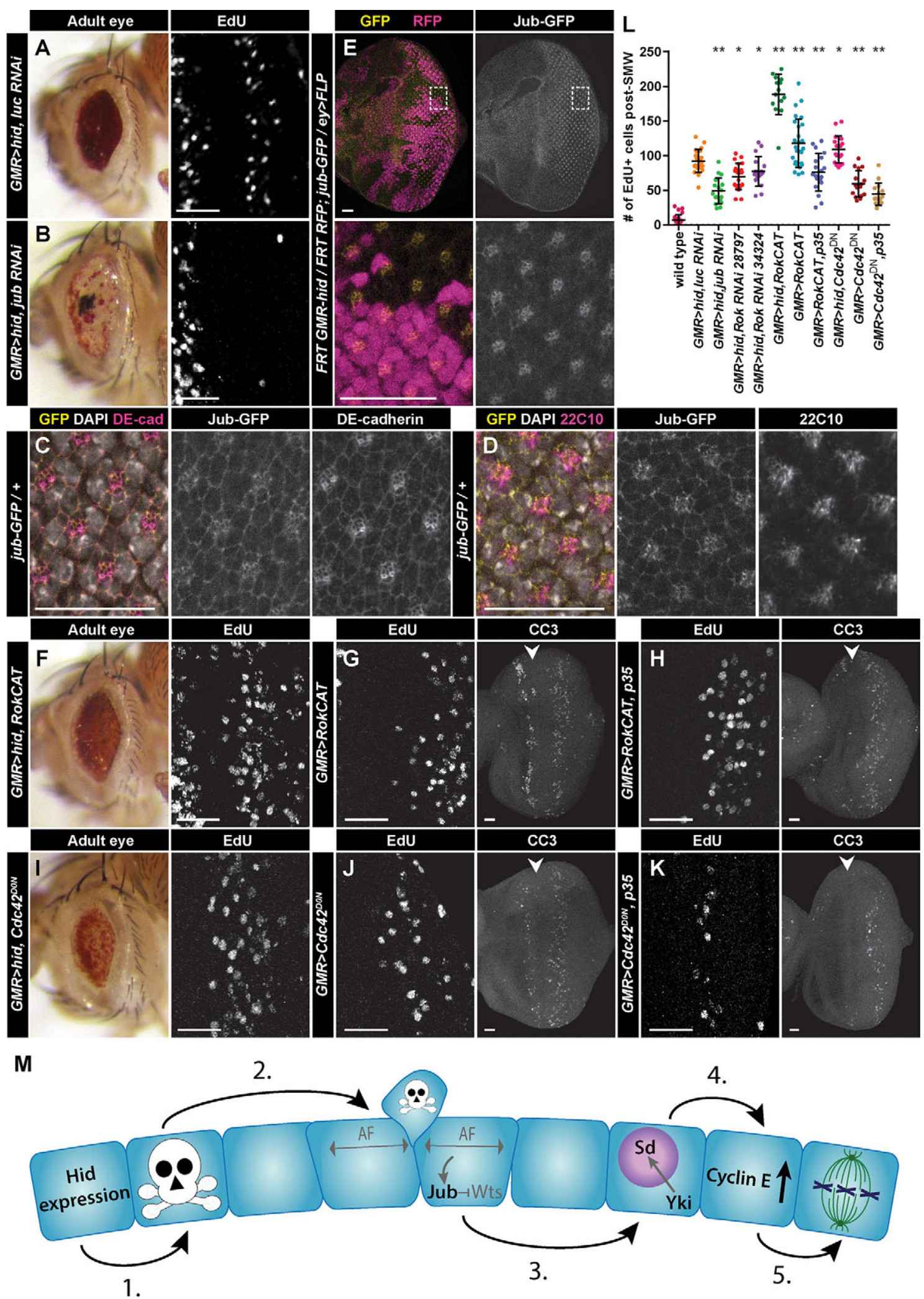

Fig. 6. See next page for legend.

re-entry and regeneration in a quiescent cell population are largely unknown, in part because many studies of regeneration have been performed in proliferative tissues. Here, we report the results from a genetic screen identifying regulators of tissue damage-induced cell cycle re-entry of a quiescent cell population in Drosophila.
Scalloped and Yorkie regulate compensatory proliferation in eye imaginal discs

We found that $\mathrm{Sd}$ and Yki are required for quiescent cells in the eye imaginal disc to enter the cell cycle in response to tissue damage. Specifically, Sd and Yki are required for Cyclin E accumulation 
Fig. 6. Jub and cellular tension regulate compensatory proliferation. $(A, B, F, I)$ Adult eyes (left panels) and high magnification of post-furrow EdU staining (right panels) of the indicated genotypes. (C,D) Jub-GFP (yellow) colocalizes with DE-cadherin (magenta; C) and Futsch/22C10, a marker of neuronal membranes (magenta; D), at the apical surface of post-furrow eye discs. (E) Clones of GMR-hid (no marker) and wild-type (RFP ${ }^{+}$, magenta) cells expressing Jub-GFP (yellow). Boxes in top panels indicate area of magnification shown in bottom panels. $(\mathrm{G}, \mathrm{H}, \mathrm{J}, \mathrm{K})$ High magnification of postfurrow EdU staining (left panels) and CC3 staining (right panels) of the indicated genotypes. Arrowheads mark the MF. GMR>RokCAT (G) and $G M R>C d c 42^{D N}(\mathrm{~J})$ induce CC3-positive apoptotic cells posterior to the MF. Interestingly, apoptotic cells are also observed anterior to the furrow in these discs. The mechanism triggering this apoptosis is unknown, but its absence in $G M R>R o k C A T, p 35(\mathrm{H})$ or $G M R>C d c 42^{D N}, p 35(\mathrm{~K})$ discs suggests the anterior induction of apoptosis in GMR $>$ RokCAT and $G M R>C d c 42^{D N}$ discs is dependent on apoptosis posterior to the furrow. CC3 staining persists posterior to the furrow after $\mathrm{p} 35$ expression $(\mathrm{H}, \mathrm{K})$ because undead cells express noncleaved-caspase epitopes of the anti-CC3 antibodies (Fan and Bergmann, 2010). (L) Quantification of CP in the indicated genotypes. GMR>hid, Gal4 genotypes were compared with $G M R>h i d$, luc $R N A i$, whereas $G M R>G a l 4$ genotypes were compared with wild type $\left(w^{1118}\right)$. Each circle represents the number of cells counted for a single disc, and bars represent mean and one standard deviation. For each genotype, $n \geq 14$ discs. ${ }^{*} P \leq 0.02,{ }^{*} P \leq 1 \times 10^{-7}$. (M) Model for induction of CP in a quiescent epithelium. Text in gray indicates proposed possibilities that have not formally been observed in this study. (1) Hid expression/tissue damage induces apoptosis in a subset of cells. (2) Basal extrusion of apoptotic cells induces apoptotic force (AF), which activates Jub, leading to Wts inhibition. (3) Wts inhibition results in Yki translocating to the nucleus and acting as a transcriptional co-activator for Sd. (4) Sd/Yki induce high levels of Cyclin E, which induces cell cycle re-entry (5). Anterior is oriented to the left. Scale bars: $20 \mu \mathrm{m}$.

following damage, presumably through transcriptional control of Cyclin E. Initial experiments suggested that Yki and Sd act together to drive gene transcription, as $\mathrm{Sd}$ is required for overgrowth and target gene induction following Yki overexpression in the eye disc (Wu et al., 2008; Zhang et al., 2008). However, their roles during eye development are clearly distinct as $y k i$ mutant clones grow poorly in the eye disc (Huang et al., 2005) whereas $s d$ mutant clones grow normally (Zhang et al., 2008). In addition, Sd and Yki have both overlapping and unique binding sites throughout the genome, many of which are tissue dependent (Slattery et al., 2013). Curiously, mutation of $s d$ rescues growth defects in $y k i$ mutant clones, suggesting that Yki may relieve Sd-mediated gene repression (Koontz et al., 2013). Additional factors, such as Tgi, probably function as co-factors for Sd suppressor function (Koontz et al., 2013). Conversely, Sd/Yki may act synergistically with E2f1 (Nicolay et al., 2011) and GAGA factor (Trithorax-like - FlyBase) (Bayarmagnai et al., 2012; Oh et al., 2013) to drive gene expression. Although our data suggest that $\mathrm{Sd}$ activates transcription during $\mathrm{CP}$, it will be important to determine how $\mathrm{Sd} / \mathrm{Yki}$ co-factors are regulated during development and tissue damage to allow robust $\mathrm{Sd} /$ Yki target gene expression.

\section{Cyclin/Cdk regulation is a conserved mechanism for inducing regeneration}

Cyclin/Cdk regulation is essential for regeneration in many model organisms. In post-mitotic Caenorhabditis elegans muscle cells, ectopic expression of cyclins drives DNA replication and cell division (Korzelius et al., 2011). Mice mutant for the CDK inhibitor p21 (Cdkn1a - Mouse Genome Informatics) robustly regenerate lost skin in the ear, which normally does not regenerate (Bedelbaeva et al., 2010). This suggests that regulation of Cyclin/Cdk activity may confer regenerative capabilities on otherwise non-regenerative tissues.

Previous work has shown that high levels of cell cycle regulators are required drive cell cycle re-entry in post-mitotic cells (Buttitta et al., 2007). The G1-S transition is driven by a positive feedback loop between E2f1 and Cyclin E where E2f1 induces Cyclin E transcription, and Cyclin E/Cdk2 inhibits Rbf, the E2f1 inhibitor. In post-mitotic photoreceptors, high levels of Rbf and Dacapo keep $\mathrm{E} 2 \mathrm{f} 1$ and Cyclin E/Cdk2 in check, and activation of both E2f1 and Cyclin E/Cdk2 is necessary to overcome cell cycle exit (Buttitta et al., 2007). In quiescent, undifferentiated cells of the developing eye disc, high levels of ectopic Cyclin E are sufficient to overcome cell cycle arrest and drive S-phase entry (Richardson et al., 1995). Undifferentiated eye disc cells may be poised to respond to damageinduced Cyclin E expression.

Although our results suggest that $\mathrm{Sd} / \mathrm{Yki}$ induce Cyclin $\mathrm{E}$ accumulation during $\mathrm{CP}$, there are likely to be other inputs that drive cell cycle re-entry. The Hedgehog pathway transcription factor $\mathrm{Ci}$, which is required for $\mathrm{CP}$ in the post-furrow eye (Fan and Bergmann, 2008), can directly activate Cyclin $E$ transcription in the eye disc (Duman-Scheel et al., 2002). Yki and Ci also have other cell cycle targets: Yki can activate E2fl transcription in the wing disc (Goulev et al., 2008) and $\mathrm{Ci}$ induces Cyclin D expression in the eye disc (Duman-Scheel et al., 2002). We postulate that a combination of $\mathrm{Ci}$ and $\mathrm{Sd} / \mathrm{Yki}$ activity drives a Cyclin E-containing gene expression program that induces cell cycle entry and $\mathrm{CP}$.

\section{Apoptotic force may link cell death to Yki activation}

In the wing imaginal disc, Yki activity during CP is driven by Jubdependent inhibition of Hippo signaling (Sun and Irvine, 2013). Our data indicate that Jub is also required for CP in the eye, suggesting a shared mechanism for Yki activation between tissues. However, although Jub-dependent inhibition of Hippo signaling in the wing disc requires JNK signaling, we did not find a requirement for JNK signaling for CP in the eye disc. An alternative mechanism for inhibition of the Hippo pathway is tension-induced activation of Jub (Rauskolb et al., 2014). Mechanical force has also been shown to regulate activity of the mammalian Yorkie homolog Yesassociated protein (YAP) (Aragona et al., 2013) and induce cell proliferation in culture (Streichan et al., 2014). We show that expression of Rok or dominant-negative $\mathrm{Cdc} 42$, which can increase cellular tension, increases cell cycle re-entry, and we propose that cellular tension in the eye disc epithelium modulates CP.

One possible source of increased cellular tension in GMR-hid discs is from 'apoptotic force' generated by extrusion of dying cells. The phenomenon of apoptotic force has been described in Drosophila embryos, in which actin/myosin-driven constriction of dying amnioserosa cells exerts force on overlying epithelial cells to drive dorsal closure (Toyama et al., 2008). Apoptotic force in GMR-hid discs may increase cellular tension and drive Jub activation. Several observations are consistent with a model that physical extrusion of apoptotic cells is required to generate the force necessary to induce CP. LGMR-hid, which induces less apoptosis than does GMR-hid and presumably less apoptotic force, does not induce CP. Additionally, the apoptotic-inhibitor p35, which blocks basal extrusion of apoptotic cells, inhibits $\mathrm{CP}$ in the eye disc posterior to the MF (Fan and Bergmann, 2008). Finally, GMR-hid clones exhibit S-phase entry in cells within and immediately bordering the clone (supplementary material Fig. S5; Fan and Bergmann, 2008). This observation suggests that CP could result from local changes in tension rather than a long-range signal.

\section{Conclusions}

The Hippo pathway is a well-conserved regulator of tissue growth and is modulated in processes such as regeneration and tumor growth. YAP is required for intestinal regeneration in mice 
(Cai et al., 2010) and confers regenerative ability in normally nonregenerative mouse hearts (Xin et al., 2013). Importantly, tight control of the Hippo pathway is crucial for tissue homeostasis, as YAP hyperactivity or inhibition of the Hippo pathway promotes cancer in many contexts (Johnson and Halder, 2014). Therefore, knowledge gained from studies of CP in Drosophila imaginal discs will contribute to understanding the role of Hippo signaling in mammalian regeneration and cancer.

\section{MATERIALS AND METHODS}

Mutants and transgenes

Wild-type control is $w^{1118}$ unless otherwise noted. All adult eye images are from females. Fly stocks were obtained from the Bloomington Stock Center or from colleagues (see supplementary Methods for more details), with the exception of LGMR-hid (construction described in the supplementary Methods). All lines used for the RNAi screen are from the Transgenic RNAi Project (TRiP) at Harvard Medical School, with details provided in supplementary material Table S1.

\section{Immunostaining}

Eye discs were dissected from wandering third instar larvae. Discs were incubated with $100 \mu \mathrm{g} / \mathrm{ml}$ 5-ethynyl-2'-deoxyuridine (EdU) or $1 \mathrm{mg} / \mathrm{ml}$ 5-bromo- $2^{\prime}$-deoxyuridine (BrdU) for $60 \mathrm{~min}$ and fixed for 20 min with $4 \%$ formaldehyde. EdU detection was performed with the Click-iT EdU Alexa Fluor 555 kit (Molecular Probes) according to the manufacturer's protocol Antibodies used are as follows: rabbit anti-cleaved Caspase-3 (1:200; 9661, Cell Signaling), rat anti-Elav [1:100; 7E8A10, Developmental Studies Hybridoma Bank (DSHB)], mouse anti- $\beta$ Gal $(1: 1000 ; 40-1 \mathrm{a}$, DSHB), mouse anti-Cyclin E (1:200; 8B10, a gift from H. Richardson, The Peter MacCallum Cancer Centre, Melbourne, Australia), mouse anti-Yan (1:500; 8B12H9, DSHB), rat anti-DE-cadherin (1:1000; DCAD2, DSHB), mouse anti-Futsch/22C10 (1:1000; DSHB), mouse anti-BrdU (1:200; B44, BD Biosciences), Alexa 488-conjugated goat anti-rabbit (1:500; 111-545-144, Jackson ImmunoResearch), Cy5-conjugated donkey anti-rat (1:500; 712175-153, Jackson ImmunoResearch). For CC3 staining, discs were incubated with primary antibodies for $48 \mathrm{~h}$ at $4{ }^{\circ} \mathrm{C}$ in PBS and a $2 \mathrm{~h}$ PBS$0.1 \%$ Triton X-100; $5 \%$ normal goat serum block was performed prior to applying secondary antibodies. Fluorescence in situ hybridizations were performed as previously described (Tomancak et al., 2002) with the following modifications: RNA probe was generated by $\mathrm{T} 7$ polymerase in vitro transcription in the presence of digoxigenin-11-UTP (Roche) from linearized hid cDNA (clone AT13267, BDGP). Detection was performed with peroxidase-conjugated anti-DIG (1:100; Roche) and Cy5-conjugated tyramide reagent (1:50; Perkin Elmer). See supplementary Methods for details on image quantification.

\section{Acknowledgements}

We thank the TRiP at Harvard Medical School and the Bloomington Drosophila Stock Center (National Institutes of Health P40OD018537) for stocks used in this study. Thanks to J. Jiang, D. Pan, I. Hariharan, T. T. Su, J. Poulton and J. Pearson for reagents; M. Peifer, D. Fox and F. Conlon for assistance in preparing this manuscript; and V. Boudreau for assistance with ImageJ quantification.

\section{Competing interests}

The authors declare no competing or financial interests.

\section{Author contributions}

Experiments were conceived by J.H.M. and R.J.D. Experiments were performed and data was analyzed by J.H.M. Figures were generated by J.H.M., and the manuscript was written by J.H.M. and R.J.D.

\section{Funding}

This work was funded by the National Institutes of Health National Institute of General Medical Sciences [T32-GM007092 to J.H.M. and R01-GM057859 and R01-GM058921 to R.J.D]; and the National Institutes of Health National Institute on Aging [F31-AG044957 to J.H.M.]. Deposited in PMC for release after 12 months.

\section{Supplementary material}

Supplementary material available online at

http://dev.biologists.org/lookup/suppl/doi:10.1242/dev.119339/-/DC1

\section{References}

Aragona, M., Panciera, T., Manfrin, A., Giulitti, S., Michielin, F., Elvassore, N., Dupont, S. and Piccolo, S. (2013). A mechanical checkpoint controls multicellular growth through YAP/TAZ regulation by actin-processing factors. Cell 154, 1047-1059.

Baker, N. E. and Yu, S.-Y. (2001). The EGF receptor defines domains of cell cycle progression and survival to regulate cell number in the developing Drosophila eye. Cell 104, 699-708.

Bandura, J. L., Jiang, H., Nickerson, D. W. and Edgar, B. A. (2013). The molecular chaperone $\mathrm{Hsp} 90$ is required for cell cycle exit in Drosophila melanogaster. PLoS Genet. 9, e1003835.

Bayarmagnai, B., Nicolay, B. N., Islam, A. B. M. M. K., Lopez-Bigas, N. and Frolov, M. V. (2012). Drosophila GAGA factor is required for full activation of the dE2f1-Yki/Sd transcriptional program. Cell Cycle 11, 4191-4202.

Bedelbaeva, K., Snyder, A., Gourevitch, D., Clark, L., Zhang, X.-M., Leferovich, J., Cheverud, J. M., Lieberman, P. and Heber-Katz, E. (2010). Lack of p21 expression links cell cycle control and appendage regeneration in mice. Proc. Natl. Acad. Sci. USA 107, 5845-5850.

Bergantinos, C., Corominas, M. and Serras, F. (2010). Cell death-induced regeneration in wing imaginal discs requires JNK signalling. Development 137 1169-1179.

Bilak, A., Uyetake, L. and Su, T. T. (2014). Dying cells protect survivors from radiation-induced cell death in Drosophila. PLoS Genet. 10, e1004220.

Brennecke, J., Hipfner, D. R., Stark, A., Russell, R. B. and Cohen, S. M. (2003) bantam encodes a developmentally regulated microRNA that controls cell proliferation and regulates the proapoptotic gene hid in Drosophila. Cell 113, 25-36.

Bryant, P. J. (1971). Regeneration and duplication following operations in situ on the imaginal discs of Drosophila melanogaster. Dev. Biol. 26, 637-651.

Buttitta, L. A., Katzaroff, A. J., Perez, C. L., de la Cruz, A. and Edgar, B. A. (2007) A double-assurance mechanism controls cell cycle exit upon terminal differentiation in Drosophila. Dev. Cell 12, 631-643.

Buttitta, L. A., Katzaroff, A. J. and Edgar, B. A. (2010). A robust cell cycle control mechanism limits E2F-induced proliferation of terminally differentiated cells in vivo. J. Cell Biol. 189, 981-996.

Cai, J., Zhang, N., Zheng, Y., de Wilde, R. F., Maitra, A. and Pan, D. (2010). The Hippo signaling pathway restricts the oncogenic potential of an intestinal regeneration program. Genes Dev. 24, 2383-2388.

Cai, Y., Zheng, H., Gong, W., Che, Y. and Jiang, B. (2011). The role of hedgehog signaling pathway in liver regeneration. Hepatogastroenterology 58, 2071-2076.

Das Thakur, M., Feng, Y., Jagannathan, R., Seppa, M. J., Skeath, J. B. and Longmore, G. D. (2010). Ajuba LIM proteins are negative regulators of the Hippo signaling pathway. Curr. Biol. 20, 657-662

de Nooij, J. C. and Hariharan, I. K. (1995). Uncoupling cell fate determination from patterned cell division in the Drosophila eye. Science 270, 983-985.

Deshpande, N., Chopra, A., Rangarajan, A., Shashidhara, L. S., Rodrigues, V. and Krishna, S. (1997). The human transcription enhancer factor-1, TEF-1, can substitute for Drosophila scalloped during wingblade development. J. Biol. Chem. 272, 10664-10668.

Dominguez, M. and Casares, F. (2005). Organ specification-growth control connection: new in-sights from the Drosophila eye-antennal disc. Dev. Dyn. 232, 673-684.

Duman-Scheel, M., Weng, L., Xin, S. and Du, W. (2002). Hedgehog regulates cell growth and proliferation by inducing cyclin D and cyclin E. Nature 417, 299-304.

Duronio, R. J. and O'Farrell, P. H. (1995). Developmental control of the G1 to S transition in Drosophila: cyclin Eis a limiting downstream target of E2F. Genes Dev. 9, 1456-1468.

Fan, Y. and Bergmann, A. (2008). Distinct mechanisms of apoptosis-induced compensatory proliferation in proliferating and differentiating tissues in the Drosophila eye. Dev. Cell 14, 399-410.

Fan, Y. and Bergmann, A. (2010). The cleaved-Caspase-3 antibody is a marker of Caspase-9-like DRONC activity in Drosophila. Cell Death Differ. 17, 534-539.

Fan, Y. and Bergmann, A. (2014). Multiple mechanisms modulate distinct cellular susceptibilities toward apoptosis in the developing Drosophila eye. Dev. Cell 30 48-60

Firth, L. C. and Baker, N. E. (2005). Extracellular signals responsible for spatially regulated proliferation in the differentiating Drosophila eye. Dev. Cell 8, 541-551.

Fox, D. T. and Duronio, R. J. (2013). Endoreplication and polyploidy: insights into development and disease. Development 140, 3-12.

Frolov, M. V., Moon, N.-S. and Dyson, N. J. (2005). dDP is needed for normal cell proliferation. Mol. Cell. Biol. 25, 3027-3039.

Gibson, M. C. and Perrimon, N. (2005). Extrusion and death of DPP/BMP compromised epithelial cells in the developing Drosophila wing. Science $\mathbf{3 0 7}$ 1785-1789.

Goulev, Y., Fauny, J. D., Gonzalez-Marti, B., Flagiello, D., Silber, J. and Zider, A. (2008). SCALLOPED interacts with YORKIE, the nuclear effector of the hippo tumor-suppressor pathway in Drosophila. Curr. Biol. 18, 435-441.

Grusche, F. A., Degoutin, J. L., Richardson, H. E. and Harvey, K. F. (2011). The Salvador/Warts/Hippo pathway controls regenerative tissue growth in Drosophila melanogaster. Dev. Biol. 350, 255-266. 
Gutierrez-Avino, F. J., Ferres-Marco, D. and Dominguez, M. (2009). The position and function of the Notch-mediated eye growth organizer: the roles of JAK/STAT and four-jointed. EMBO Rep. 10, 1051-1058.

Haynie, J. L. and Bryant, P. J. (1977). The effects of X-rays on the proliferation dynamics of cells in the wing disc of Drosophila melanogaster. Dev. Genes Evol. 183, 85-100

Huang, J., Wu, S., Barrera, J., Matthews, K. and Pan, D. (2005). The Hippo signaling pathway coordinately regulates cell proliferation and apoptosis by inactivating Yorkie, the Drosophila Homolog of YAP. Cell 122, 421-434.

Huh, J. R., Guo, M. and Hay, B. A. (2004). Compensatory proliferation induced by cell death in the Drosophila wing disc requires activity of the apical cell death caspase Dronc in a nonapoptotic role. Curr. Biol. 14, 1262-1266.

Johnson, R. and Halder, G. (2014). The two faces of Hippo: targeting the Hippo pathway for regenerative medicine and cancer treatment. Nat. Rev. Drug Discov. 13, 63-79.

Kawakami, Y., Rodriguez Esteban, C., Raya, M., Kawakami, H., Marti, M., Dubova, I. and Izpisua Belmonte, J. C. (2006). Wnt/beta-catenin signaling regulates vertebrate limb regeneration. Genes Dev. 20, 3232-3237.

Knoblich, J. A., Sauer, K., Jones, L., Richardson, H., Saint, R. and Lehner, C. F. (1994). Cyclin E controls S phase progression and its down-regulation during Drosophila embryogenesis is required for the arrest of cell proliferation. Cell 77 , 107-120.

Koontz, L. M., Liu-Chittenden, Y., Yin, F., Zheng, Y., Yu, J., Huang, B., Chen, Q. Wu, S. and Pan, D. (2013). The Hippo effector Yorkie controls normal tissue growth by antagonizing scalloped-mediated default repression. Dev. Cell 25, 388-401.

Korzelius, J., The, I., Ruijtenberg, S., Prinsen, M. B. W., Portegijs, V., Middelkoop, T. C., Groot Koerkamp, M. J., Holstege, F. C. P., Boxem, M. and van den Heuvel, S. (2011). Caenorhabditis elegans cyclin D/CDK4 and cyclin E/CDK2 induce distinct cell cycle re-entry programs in differentiated muscle cells. PLoS Genet. 7, e1002362.

Li, F., Huang, Q., Chen, J., Peng, Y., Roop, D. R., Bedford, J. S. and Li, C. Y. (2010). Apoptotic cells activate the "phoenix rising" pathway to promote wound healing and tissue regeneration. Sci. Signal. 3, ra13.

Moberg, K. H., Bell, D. W., Wahrer, D. C. R., Haber, D. A. and Hariharan, I. K. (2001). Archipelago regulates Cyclin E levels in Drosophila and is mutated in human cancer cell lines. Nature 413, 311-316.

Mollereau, B., Perez-Garijo, A., Bergmann, A., Miura, M., Gerlitz, O., Ryoo, H. D., Steller, H. and Morata, G. (2013). Compensatory proliferation and apoptosisinduced proliferation: a need for clarification. Cell Death Differ. 20, 181

Morgan, T. H. (1901). Regeneration, Vol. VII. Norwood, MA: Norwood Press.

Nicolay, B. N., Bayarmagnai, B., Islam, A. B. M. M. K., Lopez-Bigas, N. and Frolov, M. V. (2011). Cooperation between dE2F1 and Yki/Sd defines a distinc transcriptional program necessary to bypass cell cycle exit. Genes Dev. 25 , 323-335

Oh, H. and Irvine, K. D. (2008). In vivo regulation of Yorkie phosphorylation and localization. Development 135, 1081-1088.

Oh, H., Slattery, M., Ma, L., Crofts, A., White, K. P., Mann, R. S. and Irvine, K. D. (2013). Genome-wide association of Yorkie with chromatin and chromatinremodeling complexes. Cell Rep. 3, 309-318.

Peng, H. W., Slattery, M. and Mann, R. S. (2009). Transcription factor choice in the Hippo signaling pathway: homothorax and yorkie regulation of the microRNA bantam in the progenitor domain of the Drosophila eye imaginal disc. Genes Dev. 23, 2307-2319.

Perez-Garijo, A., Martin, F. A. and Morata, G. (2004). Caspase inhibition during apoptosis causes abnormal signalling and developmental aberrations in Drosophila. Development 131, 5591-5598.

Perez-Garijo, A., Martin, F. A., Struhl, G. and Morata, G. (2005). Dpp signaling and the induction of neoplastic tumors by caspase-inhibited apoptotic cells in Drosophila. Proc. Natl. Acad. Sci. USA 102, 17664-17669

Perez-Garijo, A., Fuchs, Y. and Steller, H. (2013). Apoptotic cells can induce nonautonomous apoptosis through the TNF pathway. Elife 2, e01004

Pfreundt, U., James, D. P., Tweedie, S., Wilson, D., Teichmann, S. A. and Adryan, B. (2010). FlyTF: improved annotation and enhanced functionality of the Drosophila transcription factor database. Nucleic Acids Res. 38, D443-D447.

Poss, K. D., Wilson, L. G. and Keating, M. T. (2002). Heart regeneration in zebrafish. Science 298, 2188-2190.

Rauskolb, C., Sun, S., Sun, G., Pan, Y. and Irvine, K. D. (2014). Cytoskeleta tension inhibits Hippo signaling through an Ajuba-Warts complex. Cell 158 143-156

Richardson, H., Okeefe, L. V., Marty, T. and Saint, R. (1995). Ectopic cyclin E expression induces premature entry into $S$ phase and disrupts pattern formation in the Drosophila eye imaginal disc. Development 121, 3371-3379.

Ruggiero, R., Kale, A., Thomas, B. and Baker, N. E. (2012). Mitosis in neurons: Roughex and APC/C maintain cell cycle exit to prevent cytokinetic and axonal defects in Drosophila photoreceptor neurons. PLoS Genet. 8, e1003049.
Ryoo, H. D., Gorenc, T. and Steller, H. (2004). Apoptotic cells can induce compensatory cell proliferation through the JNK and the Wingless signaling pathways. Dev. Cell 7, 491-501.

Shen, J. and Dahmann, C. (2005). Extrusion of cells with inappropriate Dpp signaling from Drosophila wing disc epithelia. Science 307, 1789-1790.

Sigal, S. H., Rajvanshi, P., Gorla, G. R., Sokhi, R. P., Saxena, R., Gebhard, D. R. Jr, Reid, L. M. and Gupta, S. (1999). Partial hepatectomy-induced polyploidy attenuates hepatocyte replication and activates cell aging events. Am. J. Physiol. 276, G1260-G1272.

Simmonds, A. J., Liu, X., Soanes, K. H., Krause, H. M., Irvine, K. D. and Bell, J. B. (1998). Molecular interactions between Vestigial and Scalloped promote wing formation in Drosophila. Genes Dev. 12, 3815-3820.

Slattery, M., Voutev, R., Ma, L., Nègre, N., White, K. P. and Mann, R. S. (2013) Divergent transcriptional regulatory logic at the intersection of tissue growth and developmental patterning. PLoS Genet. 9, e1003753.

Streichan, S. J., Hoerner, C. R., Schneidt, T., Holzer, D. and Hufnagel, L. (2014) Spatial constraints control cell proliferation in tissues. Proc. Natl. Acad. Sci. USA 111, 5586-5591

Sun, G. and Irvine, K. D. (2011). Regulation of Hippo signaling by Jun kinase signaling during compensatory cell proliferation and regeneration, and in neoplastic tumors. Dev. Biol. 350, 139-151.

Sun, G. and Irvine, K. D. (2013). Ajuba family proteins link JNK to Hippo signaling. Sci. Signal. 6, ra81.

Tamori, Y. and Deng, W.-M. (2013). Tissue repair through cell competition and compensatory cellular hypertrophy in postmitotic epithelia. Dev. Cell 25, 350-363. Thompson, B. J. and Cohen, S. M. (2006). The Hippo pathway regulates the bantam microRNA to control cell proliferation and apoptosis in Drosophila. Cell 126, 767-774.

Tomancak, P., Beaton, A., Weiszmann, R., Kwan, E., Shu, S., Lewis, S. E., Richards, S., Ashburner, M., Hartenstein, V., Celniker, S. E. et al. (2002) Systematic determination of patterns of gene expression during Drosophila embryogenesis. Genome Biol. 3, research0088.14.

Toyama, Y., Peralta, X. G., Wells, A. R., Kiehart, D. P. and Edwards, G. S. (2008) Apoptotic force and tissue dynamics during Drosophila embryogenesis. Science 321, 1683-1686

Tseng, A.-S., Adams, D. S., Qiu, D., Koustubhan, P. and Levin, M. (2007) Apoptosis is required during early stages of tail regeneration in Xenopus laevis. Dev. Biol. 301, 62-69.

Verghese, S., Bedi, S. and Kango-Singh, M. (2012). Hippo signalling controls Dronc activity to regulate organ size in Drosophila. Cell Death Differ. 19 1664-1676.

Warner, S. J. and Longmore, G. D. (2009). Cdc42 antagonizes Rho1 activity at adherens junctions to limit epithelial cell apical tension. J. Cell. Biol. 187, 119-133. Warner, S. J., Yashiro, H. and Longmore, G. D. (2010). The Cdc42/Par6/aPKC polarity complex regulates apoptosis-induced compensatory proliferation in epithelia. Curr. Biol. 20, 677-686

Wenemoser, D. and Reddien, P. W. (2010). Planarian regeneration involves distinct stem cell responses to wounds and tissue absence. Dev. Biol. 344 979-991.

Wernet, M. F., Labhart, T., Baumann, F., Mazzoni, E. O., Pichaud, F. and Desplan, C. (2003). Homothorax switches function of Drosophila photoreceptors from color to polarized light sensors. Cell 115, 267-279.

Winter, C. G., Wang, B., Ballew, A., Royou, A., Karess, R., Axelrod, J. D. and Luo, L. (2001). Drosophila Rho-associated kinase (Drok) links Frizzled-mediated planar cell polarity signaling to the actin cytoskeleton. Cell 105, 81-91.

Worley, M. I., Setiawan, L. and Hariharan, I. K. (2012). Regeneration and transdetermination in Drosophila imaginal discs. Annu. Rev. Genet. 46, 289-310. Wu, S., Liu, Y., Zheng, Y., Dong, J. and Pan, D. (2008). The TEAD/TEF family protein Scalloped mediates transcriptional output of the Hippo growth-regulatory pathway. Dev. Cell 14, 388-398.

Wuestefeld, T., Pesic, M., Rudalska, R., Dauch, D., Longerich, T., Kang, T.-W., Yevsa, T., Heinzmann, F., Hoenicke, L., Hohmeyer, A. et al. (2013). A direct in vivo RNAi screen identifies MKK4 as a key regulator of liver regeneration. Cell 153 389-401.

Xin, M., Kim, Y., Sutherland, L. B., Murakami, M., Qi, X., McAnally, J., Porrello, E. R., Mahmoud, A. I., Tan, W., Shelton, J. M. et al. (2013). Hippo pathway effector Yap promotes cardiac regeneration. Proc. Natl. Acad. Sci. USA 110 13839-13844

Yu, F.-X. and Guan, K.-L. (2013). The Hippo pathway: regulators and regulations. Genes Dev. 27, 355-371.

Zhang, L., Ren, F., Zhang, Q., Chen, Y., Wang, B. and Jiang, J. (2008). The TEAD/ TEF family of transcription factor Scalloped mediates Hippo signaling in organ size control. Dev. Cell 14, 377-387.

Zhang, H.-M., Chen, H., Liu, W., Liu, H., Gong, J., Wang, H. and Guo, A.-Y. (2012). AnimalTFDB: a comprehensive animal transcription factor database. Nucleic Acids Res. 40, D144-D149. 